\title{
Anabases
}

ANABASES Traditions et réceptions de l'Antiquité

$21 \mid 2015$

Varia

\section{Le roman historique est-il archéocompatible ?}

\section{Claude Aziza}

\section{OpenEdition}

Journals

Édition électronique

URL : http://journals.openedition.org/anabases/5320

DOI : 10.4000/anabases. 5320

ISSN : 2256-9421

\section{Éditeur}

E.R.A.S.M.E.

\section{Édition imprimée}

Date de publication : 1 avril 2015

Pagination : 181-194

ISSN : 1774-4296

\section{Référence électronique}

Claude Aziza, «Le roman historique est-il archéocompatible ? », Anabases [En ligne], 21 | 2015, mis en ligne le 01 avril 2018, consulté le 21 octobre 2019. URL : http://journals.openedition.org/anabases/ 5320 ; DOI : 10.4000/anabases.5320 


\section{Le roman historique est-il archéocompatible ${ }^{1}$ ?}

Claude Azıza

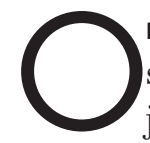

n voudra bien pardonner ici un terme volontairement ludique pour un sujet qui risque de l'être moins, à savoir examiner les liens entre la toute jeune archéologie et ce nouveau genre littéraire qu'est le roman, qu’il soit historique ou autre. Tous deux sont nés à la fin du XvıI ${ }^{\mathrm{e}}$ siècle, ont prospéré tout au long du siècle suivant, ont fait les beaux jours du $\mathrm{xx}^{\mathrm{e}}$ et se portent à merveille aujourd'hui. Ce type de roman a-t-il une dette envers cette jeune science? Les chantiers de fouilles ont-ils inspiré des romans historiques? Tentative de réponse en six romans et autant de nouvelles.

\section{Les Derniers Jours de Pompéi ou l'Antiquité ressuscitée (1834)}

Peu de temps avant Les Martyrs (I809), en I804, Chateaubriand, secrétaire d'ambassade à Rome depuis I8o3, est allé voir Pompéi. Il en a retiré une impression, très en avance pour son temps : “Il faut laisser les choses, écrit-il dans son Voyage en Italie, dans l'endroit où on les trouve. Ne serait-ce pas là le plus merveilleux musée de la terre? Une ville romaine conservée tout entière, comme si ses habitants venaient d'en sortir un quart d'heure auparavant? „ Un autre écrivain ira encore plus loin, E.G.Bulwer-Lytton (1803-I873).

Car, en ce début de siècle, pour ressusciter la vie des siècles passés, il fallait à la fois se faire historien et « antiquaire », terme qui englobera au siècle suivant à la

1 Ce texte est la version abrégée d'une conférence faite le ı8octobre 20I4, au Musée archéologique de Nîmes, dans le cadre d'un cycle sur l'Archéologie. 
fois l'historien et l'archéologue des siècles passés. Walter Scott survint alors, qui commença à vouloir faire revivre cette Écosse, d'où il était originaire. Ses romans furent portés aux nues dans toute l'Europe. Mais, bien qu'elle ne touchât en rien à l'Antiquité, l'œuvre romanesque de Scott allait jeter les bases d'une nouvelle vision historique qui inspirera tout le siècle, mais, au premier chef, le plus grand des disciples du romancier, Edward George Bulwer-Lytton, l'immortel auteur des Derniers Jours de Pompéi.

Edward George Bulwer, qui deviendra plus tard (en i866) baron Lytton de Knebworth, reste aujourd'hui, l'homme d'un seul roman mais en réalité ses œuvres dépassent la centaine. Poète couronné, auteur dramatique à succès, romancier doué, homme d'État, grand voyageur, il a été tout cela.

Mais revenons à cet hiver I832-I833 qui voit notre jeune romancier, marié depuis peu, partir en Campanie avec une épouse pour laquelle il n'éprouve pas une grande passion. Laquelle goûte peu aux charmes d'une région alors insalubre et préfère les agréments napolitains aux rêveries sur les cités disparues. Va-t-elle peu ou prou rendre jaloux son tiède époux? Va-t-il retrouver au pied du Vésuve, alors éteint, le souvenir d'un amour de jeunesse déçu? Peu importe. Le cœur a ses raisons qui nourrissent la création littéraire: c'est l'essentiel. Il faut imaginer notre jeune écrivain romantique errant, seul, le cœur en écharpe, dans une cité alors ouverte à tous, devenue, depuis un petit demi-siècle, le lieu de passage obligé de toute l'Europe. Le Vésuve prend part à la fête: il gronde en I794, perd encore deux cent mètres de haut mais se rattrape en dévorant Torre Del Greco; il s'épanche encore en I822.

Mais rien n'y fait: écrivains et princes affluent: Goethe, puis Walter Scott, en compagnie de William Gell, auteur d'ouvrages savants sur Pompéi, à qui BulwerLytton - qui fut son hôte à Naples - dédicacera son roman en lui témoignant toute son admiration. C'est sans doute par lui qu'il connut les réactions de Scott visitant Pompéi : «La cité de la Mort! La cité de la Mort.» On peut imaginer que cette réflexion lui a donné le désir de refaire de Pompéi une cité de la Vie. Il arrivait d'ailleurs au bon moment; les découvertes succédaient aux découvertes: en I8Io, des amulettes trouvées dans le temple d'Isis, un des hauts lieux du roman, en I8I5, l'amphithéâtre est dégagé, en i824, c'est le tour du temple de la Fortune Auguste et, un an plus tard, c'est la découverte d'une maison que l'on baptise «Maison du poète tragique». Ce sera la demeure de Glaucos, le héros du roman. En I83I, encore, on découvre la superbe mosaïque d'“Alexandre à Issus ».

Le secret du roman se trouve alors dans la préface: «Repeupler une fois encore ces rues désertes, restaurer ces ruines élégantes et réanimer des ossements encore cachés $(\mathrm{au})$ regard, traversant un gouffre de dix-huit siècles et éveillant à une seconde existence la cité de la Mort!» Il s'agit non seulement de rivaliser avec le Maître mais, bien plus, de le surpasser. Mais la tâche est difficile, «car, nous sommes sans lien domestique ou familier avec l'âge classique [...]; et ces choses, 
liées à des souvenirs d'étude imposées comme besogne et non cultivées comme plaisir, nous furent rendues encore plus usées par les pédanteries scolastiques qui en premier lieu nous les firent connaître. »

Les Derniers Jours de Pompéi paraissent en I834, le romancier a choisi une fiction directement liée à l'histoire mais il a mis l'érudition à son service. Certes il sait se montrer latiniste, archéologue, historien, moraliste, philosophe. Mais il refuse la pédanterie, adopte un langage vivant, n’hésite pas devant les situations mélodramatiques et se veut poète romantique. Pompéi, sous les auspices de Scott et de Goethe, est redevenue une cité vivante que l'on peut visiter avec pour guide le roman de Bulwer-Lytton. Bien plus, au-delà d'un fatras romantique, un peu dépassé aujourd'hui, on y trouvera une mine de renseignements, dont certains donnent à voir des étapes aujourd'hui disparues de la résurrection de la cité. Avec les ans, le roman s'est fait à la fois guide archéologique, témoin d'un temps disparu, sans perdre sa force et son charme, fût-il parfois suranné.

Avant Bulwer, il y avait eu, à Pompéi, Goethe, le II mars I787 que l'exiguïté de la cité surprend et qui regrette, lors de sa visite à Herculanum, le lendemain que “les fouilles n'aient pas été faites méthodiquement par des mineurs allemands.» Il est vrai que trois ans plus tôt, en I784, Winckelmann comptait dans les quelque cinquante travailleurs attribués aux fouilles - et dont seuls huit étaient au travail lors de sa visite! - «les esclaves d'Alger et de Tunis »...

Après Chateaubriand, ce seront Madame de Staël, en i8o5, Lamartine, en I8II, Stendhal, en i8ı7. Après lui Nerval, en ı834, l'année même de la parution du roman, puis, de retour d'Orient en i843. Pompéi lui inspire un curieux texte, Le Temple d'Isis, souvenir de Pompéi, qui deviendra Isis (1845), une nouvelle reprise ensuite dans le recueil, Les Filles du feu (I854). Son ami Gautier, visite la cité en i85o. Cette visite lui inspirera, en ı852, une nouvelle, Arria Marcella. Flaubert, le suivra d'une année; Taine viendra en i864, Freud, enfin, en igo2.

Reste Alexandre Dumas qui suit d'une année Bulwer-Lytton, lui aussi à la recherche d'une Antiquité plus vivante que celle de ses souvenirs d'études.

\section{Acté ou l'Antiquité flamboyante (1839)}

“Quand je suis bien las, bien fatigué, bien abruti, je trouve un prétexte pour aller à Rome et j'y vais. Quand je ne trouve pas de prétexte, j'y vais encore.

- Mais que faites-vous à Rome? me demanderez-vous.

- Oh! ce que je fais à Rome, c'est bien facile à dire: je vais voir la via Appia; je vais regarder couler le Tibre; je vais m'asseoir sous une arcade du Colisée, et je dis à part moi: "Il faut pourtant que je fasse une histoire de Rome."

- Pourquoi ne la faites-vous pas alors?

- Parce qu'elle serait trop amusante; personne ne la lirait. » 
Aveu faussement naif ou vraiment roublard? Lorsque Dumas écrit ces lignes, en I86o, il a depuis longtemps compris que pour séduire avec l'Histoire, la vraie, la grande, les lecteurs, il vaut mieux avoir le sens de l'épopée ou du drame que celui de la pédagogie. Le secret? Le romancier nous le livre dans la préface de Caligula (I837), une tragédie en cinq actes et en vers: faire revivre "toute une époque inconnue, ou, qui pis est, mal connue; une époque que, arrivés à un certain âge, nous ne revoyons plus qu'à travers les souvenirs fastidieux du collège [...]. Ajoutez à cela que l'Antiquité [...] était tombée dans un si merveilleux discrédit, que l'ennui qu'elle traînait à sa suite était devenu proverbial [...]. Je partis pour l'Italie, afin de voir Rome; car, ne pouvant étudier le cadavre, je voulais au moins visiter le tombeau. Je restais deux mois dans la ville aux sept collines, visitant le Vatican le jour, et la nuit le Colisée; mais après avoir tout rebâti dans ma pensée, depuis les prisons Mamertines jusqu'aux bains de Titus, je m'aperçus que je n'avais qu'une face du Janus antique; face grave et sévère [...]. C'était Naples, la belle esclave grecque, qui devait m'offrir ce second visage, voilé, pour nos grands maîtres, sous la lave d'Herculanum et la cendre de Pompéi. »

Arrivé le rg juin en Italie, Dumas part donc pour Naples le 2 août, quasiment sur les traces de Bulwer-Lytton. Il y restera jusqu'au 23, après avoir longuement visité la ville, Pouzzoles, le Vésuve, Herculanum et Pompéi où il décide de séjourner, mais à sa façon: «Je descendis dans les souterrains de Résina, je m’établis dans la maison du Faune; pendant huit jours je vécus, m'éveillant et m'endormant dans une habitation romaine, touchant du doigt l'Antiquité.» Faut-il le croire? Avec ce diable d'homme, tout est possible!

Cet amour pour Pompéi laissera chez Dumas une trace si profonde, cet émerveillement sera si durable, qu'en septembre 1860 - il vient de terminer son second roman sur Rome, les Mémoires d'Horace - il demande à Garibaldi, le nouveau maître du royaume de Naples, en récompense de ses services - fourniture d'armes - ... la direction des musées de Naples et des fouilles de Pompéi. Et il l'obtient. Pour peu de mois, il est vrai : Fiorelli, l'inventeur des moulages, lui succédera en décembre.

Comment est donc venu à Dumas le désir de parler de l'Antiquité? Sans doute pour les mêmes raisons que Bulwer-Lytton: dans les deux cas, il s'agit d'une Antiquité qui a pris les couleurs romantiques: un amour fou, auquel l'héroïne - tendre et touchante comme il se doit - succombe immédiatement. Elle y était prédestinée. Mais chez l'un, après avoir vaincu la folie des hommes et la rage de la nature, il triomphera. Alors que chez l'autre cet amour est sans espoir. Bien plus, il entrâ̂nera l'innocente Acté dans le péché au point qu'elle devra trouver dans la religion chrétienne - telle une nouvelle Marie-Madeleine - son salut par le rachat. Car chez nos deux romanciers, le christianisme n'est jamais bien loin. Parfois, comme chez le premier, en faisant fi de toute vérité historique. 
Mais la radicale nouveauté d'Acté se trouve dans le portrait que le romancier a donné de Néron. La réputation sulfureuse de celui que les Pères de l'Eglise, avec une complaisance sans cesse renouvelée, ont nommé “l'Antéchrist», n’a jamais cessé de croître et d'embellir. Or Dumas, en l'absence de toute documentation scientifique sérieuse sur le personnage, en donne - comme beaucoup d'historiens contemporains - un portrait somme toute positif.

Le roman est construit comme une tragédie en cinq actes : les jeux de Corinthe, la cour de Néron, les catacombes, les noces impériales, la mort du tyran. Mais si Dumas ne résiste pas à décrire les jeux du cirque, bizarrement, il bâcle en quelques lignes, la tarte à la crème - si l'on ose dire - de tous les romans qui suivront: l'incendie de Rome de 64. Le romancier refuse la description pour la description: son héroïne n'est pas mêlée à l'incendie dont le complaisant tableau n'obéirait à aucune nécessité dramatique.

Si, avec Acté, la littérature française entrait, avec toute la pompe du triomphe romain, dans le roman à l'antique, l'influence de Chateaubriand restait trop grande pour qu'on puisse abandonner si vite l'Antiquité chrétienne. Il aura un successeur de talent en la personne du cardinal Wiseman, qui écrit en i854 Fabiola ou l'église des catacombes.

\section{Fabiola ou l'Antiquité chrétienne (1854)}

La vie et la carrière du romancier, né à Séville en I802, la même année que Dumas et Hugo, ne manquent pas d'intérêt. Brillantes études classiques, docteur en théologie, linguiste confirmé, spécialiste de l'hébreu et du syriaque. Ordonné prêtre en I825, professeur d'hébreu et de syro-chaldéen au collège où il avait fait ses études, il commence une belle carrière à la fois scientifique et littéraire. Évêque en i840, vicaire apostolique de Rome en I85o, nommé cardinal la même année par le pape Pie IX.

Après la publication d'Essais en I853, Wiseman songe à une façon agréable et efficace de raconter les débuts du christianisme. Il y travaillait par à-coups déjà. Profitant d'une maladie et d'un lent voyage à Rome, il compose Fabiola dont le succès est immédiat. Il sera durable. À sa mort, en I865, il aura droit à des funérailles nationales.

Une lecture rapide du roman y verrait un ouvrage de patronage, relevé des structures littéraires nouvelles. Au mélodrame il emprunte son ton larmoyant, ses accents insupportable (du moins aujourd'hui), ses ressorts usés. Au feuilleton, ses coups de théâtre, ses reconnaissances subites, ses familles déchirées dont, ici, la fille est déjà “chrétienne de cœur», comme disait Tertullien en parlant de Pilate, tandis que le père est un Romain de la vieille espèce. Pourtant le roman puise aux meilleures sources de la recherche historique du siècle. Wiseman, antiquisant confirmé, est au fait de la toute nouvelle archéologie chrétienne, illustrée par 
Giovanni Battista de Rossi. Ainsi Fabiola peut, à la limite, se présenter comme un manuel d'archéologie chrétienne à l'usage des jeunes lecteurs du xix ${ }^{\mathrm{e}}$ siècle, des adultes du xx ${ }^{\mathrm{e}}$ siècle et des savants du xxi ${ }^{\mathrm{e}}$ siècle...

Le hasard fait que parait en cette même année i854, cette somme monumentale qu'est l'Histoire romaine de Theodor Mommsen (I8I7-Igo3). Quant aux résultats des recherches de Rossi, ils ont certes été publiés après le roman (en I86I, ses Inscriptiones Christianae Urbis Romae, suivies par le Dictionnaire des Antiquités chrétiennes de Martigny, en I865) mais ses travaux ne sont pas restés inconnus de Wiseman qui les cite sans cesse dans ses notes. Enfin, il faudrait pouvoir démontrer - mais la chose est difficile - que le roman de Wiseman est aussi un jalon important dans l'histoire de l'Église au $\mathrm{xxx}^{\mathrm{e}}$ siècle. On ne peut que signaler quelques éléments qu'on voudrait signifiants. Il est clair que le public, déjà fasciné par les découvertes de l'archéologie païenne, notamment à Pompéi, a goûté aux charmes de celle chrétienne. Un exemple, entre cent, le plus spectaculaire peutêtre: c'est en 1854 - encore une coïncidence - que fut mise à jour la «crypte des papes ", une chambre souterraine qui abrite les dépouilles de nombre de papes du III ${ }^{\mathrm{e}}$ siècle, la plupart morts en martyrs. Tout près se trouve le cubiculum qui abritait jusqu'au $\mathrm{x}^{\mathrm{e}}$ siècle, la tombe de sainte Cécile, la Caecilia du roman.

Tout comme pour Pompéi, le public sera fasciné par ces mondes souterrains au point de s'en faire une idée aussi romantique qu'inexacte. S'il fallait une clé pour expliquer le succès de Fabiola (voire celui de ses épigones), nous la rechercherions volontiers dans le roman de Bulwer. Ici et là un monde enseveli que fait redécouvrir l'archéologie, un monde souterrain, une descente aux enfers, païens ou chrétien, peu importe. Une société paisible tournée vers l'amour - païen ou divin - qu'une catastrophe imminente, volcan ou persécution, menace de détruire. Quant au portrait que fait Wiseman des tyrans persécuteurs que sont Dioclétien et Galère, il emprunte des traits à celui - moins traditionnel - du héros du roman de Dumas.

Mais, il est temps de revenir en France pour découvrir d'autres horizons antiques, du haut des pyramides ou dans les ruines de Carthage. Changement de décor!

\section{Le Roman de la momie ou l'Antiquité refuge (1857)}

Qui mieux que Baudelaire a pu comprendre Gautier (I8II-I872) qui fut son maitre et son modèle? «Le goût du beau, écrit-il dans L'Art romantique, est pour lui un fatum. [...] La muse de Théophile Gautier habite un monde plus éthéré. [...] Elle aime ressusciter les villes défuntes, et faire redire aux morts rajeunis leurs passions interrompues. [...] Mais ce qu'elle aime surtout, c'est, debout sur les rivages parfumés de la mer Intérieure, nous raconter avec sa parole d'or cette gloire qui fut la Grèce et cette grandeur qui fut Rome...» 
Pour Gautier dont la sensibilité tient à la fois du peintre qu'il aurait voulu être et du poète qu'il refusa toujours d'éteindre en lui, malgré les vicissitudes d'une vie tout entière de labeur, pour Gautier, donc, l'Antiquité, plus que ce Moyen Âge - mythique - où se réfugièrent ses pairs, est, d'abord, un monde de grâces, de charmes et de beauté. Cette beauté sous toutes ses formes, les récits, roman et nouvelles, où Gautier évoque le monde antique permettent d'en saisir les multiples aspects.

Qu'y a-t-il de commun, en effet, entre l'Égypte des pharaons du Roman de la momie (1857) et l'Athènes de Périclès de La Chaîne d'or ou l'amant partagé (1837)? Entre la mythique Lydie du Roi Candaule (I844) et la troublante Pompéi d'Arria Marcella (1852)? Entre la Cléopâtre d'Une nuit de Cléopâtre (1838) et l'Omphale d'Omphale, ou la tapisserie amoureuse (1834)? Peu de choses, sinon que toutes, peu ou prou, sont associées à des contrées fabuleuses où le rêve peut prendre son essor sans entraves et où l'imagination se donne libre cours dans une luxuriance de formes et de couleurs.

Mais d'où vient l'amour de l'Égypte chez Gautier? S'il est lié aux découvertes archéologiques de son temps, on le fera remonter à cette année I8I7 où Belzoni découvre l'hypogée de Séti ${ }^{\mathrm{Ier}}$ qu'il reconstitue à Paris en I823. À moins que ce ne soit l'exposition de la collection Passalacqua à la galerie Vivienne, à Paris, cette même année ı826 qui voit la création, le ı6 mai, au Louvre d'une salle (ouverte au public le 6janvier ı 828) consacrée aux monuments égyptiens dont Champollion est le conservateur. Ce même Champollion, de retour en I829, d'un voyage en Égypte avec un cadeau... pharaonique: l'obélisque de Louxor et qui prononce sa leçon inaugurale d'Égyptologie le ıomai ı83ı au Collège de France, avant de mourir le 4 mars I832, laissant des Lettres écrites d'Égypte et de Nubie en I828 et I829, publiées en I833 et où l'on trouve le nom de l'héroïne du roman de Gautier, Tahoser, mais orthographié ici Thaoser.

À moins qu'il ne faille chercher du côté des “orientalistes », surtout chez Prosper Marilhat qui expose au salon de i834 une vue du Caire: Place de l'Esbekieh. Dont Gautier dira dans un article du gmars I836, repris dans la Revue des Deux Mondes le $\mathrm{I}^{\mathrm{er}}$ juillet $\mathrm{I} 848$ : “Aucun tableau ne fit sur moi une impression plus profonde et plus longtemps vibrante [...]. Je crus que je venais de reconnaître ma véritable patrie.» Ou du côté de la musique avec l'opéra de Rossini, Mö̈se ou le passage de la mer Rouge que l'opéra de Paris reprend en 1852.

Mais dans l'œuvre de l'écrivain tout commence en I840 par un pied charmant qui trouble et qui fascine celui qui le contemple. À lui en faire perdre la raison. C'est un pied de femme, bien sûr, bien conservé des outrages du temps qu'il a traversé, derrière le rempart de ses bandelettes, pour vanter les charmes vénéneux du passé et flatter les fantasmes... de son créateur. On l'aura deviné, c'est un pied de momie et Théophile Gautier, l'auteur de la nouvelle homonyme (parue en septembre 1840 dans Le Musée des familles), fait partie de ces hommes du XIx ${ }^{\mathrm{e}}$ siècle 
pour qui la femme est d'autant plus objet de désir qu'elle se présente sous la forme de morceaux choisis! Entre le pied et la momie tout entière dix-sept ans. Marqués par l'exposition de «la chambre des Rois» de Karnak à la Bibliothèque nationale en I844 et par les «Nostalgies d'obélisques» de Gautier, publiées dans la Presse du 4août i85ı. Surtout peut-être, par la publication, en i856, de l'Histoire des usages funèbres et des sépultures des peuples anciens d'Ernest Feydeau dont l'écrivain fera un compte rendu nourri le 3i octobre 1856 dans Le Moniteur universel.

On y est ou presque: du in mars au 6 mai i857 Le Roman de la momie paraît dans Le Moniteur universel, puis en librairie en avril de l'année suivante. L'Égyptomanie avait enfin son drapeau littéraire! Deux ans avant le début des travaux de creusement du futur canal de Suez. Là, se mêlent harmonieusement thèmes bibliques, l'Exode et la traversée de la mer Rouge, égyptomaniaques, la recherche archéologique, fantastiques, un amour qui traverse le temps et... oniriques, la recherche - presque platonicienne - de l'objet du désir qui permettra au Moi divisé de retrouver son unité. Roman de l'amour impossible, celui que l'on retrouvera dans toutes les nouvelles de Gautier.

Mais le retour à la réalité réservera bien des déceptions au poète quand il sera confronté en juin I867, lors de l'Exposition universelle, au démaillotage, si à la mode à l'époque, d'une momie, Nes-Khons, qui n'a que de lointains rapports avec la charmante Tahoser. Mais surtout, deux ans plus tard, lorsqu'il part, enfin, en Égypte pour l'inauguration du canal de Suez et que, victime d'une chute sur le bateau, il ne peut jouir pleinement de ce moment tant attendu. Une seule et maigre consolation: il sera logé à l'hôtel Sheppeard... place de l'Esbekieh. "On nous logeait dans notre rêve», s'écrie-t-il, avec le ravissement d'un désir enfin réalisé et la mélancolie qui en suit nécessairement sa satisfaction. Car Gautier restera toute sa vie un «animal triste post scriptum ». Merci Lucrèce...

Domaine du beau, refuge de l'idéal, l'Antiquité c'est aussi le territoire de «la rétrospective évocatrice ", pour reprendre les termes de Georges Poulet dans ses Études sur le temps humain (I949). “C'est une espèce d'évocation magique du passé, où ce que les yeux ont vu et ne reverront plus, se relève un moment de sa tombe d'oubli, et apparaît avec les couleurs d'une vie fantasmatique.» L'art peut donc par une sorte d'effet, que Gautier lui-même nomme "galvanisme» - un terme à la mode -, ressusciter les morts, si bien qu'on finit par s'intéresser aux fantômes qu'évoque la magie du poète, qu'on les prend pour les individualités historiques dont il a voulu remuer les cendres.

Ces cendres ne sont-elles point précisément celles qui recouvrirent, en 79 de notre ère, la ville de Pompéi et qui enchâssèrent dans un sarcophage de boue durcie Arria Marcella? Que le regard d'Octavien, le héros de la nouvelle de Gautier, délivrera de sa prison, tout comme celui de lord Evandale fera revivre la momie Tahoser. Ainsi l'imagination recrée un monde antique qui éveille le désir et transporte l'esprit hors du temps. C'est ce que va, à son tour, tenter Flaubert. 


\section{Salammbô ou l'Antiquité évasion (1862)}

«Peu de gens devineront combien il a fallu être triste pour entreprendre de ressusciter Carthage! C'est là une Thébaïde où le dégoût de la vie moderne m’a poussé. »C'est ainsi que Gustave Flaubert (I82I-I880) annonce, le 29 novembre I859, son projet à Ernest Feydeau, le même qui inspira - on l'a vu - Gautier. En fait, il remonte bien plus loin, à cette année I85o, où il voyage en Égypte, avec son ami Du Camp. Un “conte égyptien (lui) trotte dans la tête»: Anubis ou l'histoire d'un amour impossible. La rédaction de Madame Bovary va l'épuiser: “Cette cohabitation morale avec des bourgeois me tourne sur le cœur et m’épuise. Je sens le besoin de vivre dans des milieux plus propres [...]. Il est temps que je m'amuse [...] C'est pourquoi je me perds, tant que je peux, dans l'Antiquité.» Annoncé depuis I857, maintes fois retardé après son voyage à Carthage en I858, le roman est repris de fond en comble à partir de juillet I858.

Primitivement, il devait s'appeler Les Mercenaires, puis il se transforme en Salammbô, roman carthaginois, enfin il s'intitule Salammbô. Le nom même de l'héroïne a considérablement varié: Pyra, Pyrrha, Hanna, Salambô (avec un seul M). Avant d'aboutir au titre final dont le sens, voulu par l'écrivain par l'ajout d'un second $\mathrm{M}$ (qui oblige à prononcer SALAMM), renvoie à la fois à Emma Bovary - ce que comprendra fort bien Sainte-Beuve dans sa critique du roman - et à la formule orientale Salamm qui est une sorte de salut. C'est donc une façon de saluer Emma Bovary à laquelle Salammbô emprunte bien des traits mais en même temps de dire adieu - pour un temps du moins - à la forme réaliste romanesque qui avait été jusque-là celle de Flaubert, pour aborder un style baroque et flamboyant. Soutenu par «un travail archéologique formidable» («J'ai une indigestion de bouquins. Je rote de l'in-folio [...] Savez-vous combien je me suis ingurgité de volumes sur Carthage? Environ Ioo »).

Car beaucoup d'ouvrages ont paru depuis i833 et les travaux de C.T. Falbe, consul général du Danemark à Tunis, qui dresse une carte archéologique de Carthage pour accompagner ses Recherches sur l'emplacement de Carthage. Quatre ans plus tard on crée une “Société pour l'exploration de Carthage», dont le président, Dureau de la Malle, avait publié, en I835, des Recherches sur la topographie de Carthage. Mais c'est en I859 que commence vraiment, avec Charles-Ernest Beulé, «l'inventeur » des Propylées de l'Acropole d'Athènes, la résurrection de Carthage. Bientôt Edmond de Sainte-Marie découvre 2000 stèles puniques. Ernest Renan fait, de I86o à I86ı, une mission d'exploration en Phénicie. On peut imaginer que la création, en I885, d'un service des Antiquités à Carthage doit beaucoup à l'immense succès du roman de Flaubert.

En l'écrivant, le romancier a voulu, comme il l'écrit à Sainte-Beuve, «fixer un mirage ». Mais ce mirage, il n'a pu lui donner forme qu'en «appliquant à l’Antiquité les procédés du roman moderne». Roman historique? Roman archéologique? 
“Roman antique», le terme est de l'écrivain lui-même? Roman réaliste même? “Roman carthaginois », voire? Flaubert sait bien que «si le roman est aussi embêtant qu'un bouquin scientifique, bonsoir, il n'y a plus d'Art.» Voila pourquoi le formidable travail archéologique, théorique et pratique, sur le terrain, à Carthage, auquel s'est livré l'écrivain, ne vise pas à une vérité, impossible, du moins à l'époque, à atteindre. Il s'agit surtout de “faire vrai», de créer une “illusion» de réalité, bref d'aboutir à cet “ effet de réel », au sens où l'entendra Barthes, un siècle plus tard.

Cette Antiquité punique, dont le visage barbare tranche avec celui plus policé de la grecque ou de la romaine, se veut comme une provocation lancée à la face de la société gourmée du temps de Flaubert. Ne déclare-t-il pas lui-même: "Salammbô $\mathrm{I}^{\circ}$ embêtera les bourgeois, c'est-à-dire tout le monde; $2^{\circ}$ révoltera les nerfs et le cœur des personnes sensibles; $3^{\circ}$ irritera les archéologues; $4^{\circ}$ semblera inintelligible aux dames $; 5^{\circ}$ me fera passer pour pédéraste anthropophage? Espérons-le» (Lettre à E. Feydeau, octobre I862). Mais là ne se situe pas la scandaleuse nouveauté du roman. Les moments sadiques (la fameuse "grillade des moutards») ne font plus frémir et la version définitive a émoussé les audaces érotiques (la «baisade sous le péplos»).

C'est sur le plan de l'écriture, dans le choix du vocabulaire, dans les hardiesses de la forme, cette forme poétique appliquée au romanesque. Ajoutons que s'intéresser à “une civilisation perdue, anéantie; un pays de ruines »- les mots sont de Sainte-Beuve -, c'est relever un défi: “Ce n'est pas une petite ambition que de vouloir entrer dans le cœur des hommes quand ces hommes ont vécu il y a plus de deux mille ans et dans une civilisation qui n'a rien d'analogue avec la nôtre.» On croirait lire la préface des Derniers Jours de Pompéi ou retrouver les mots de l'ami Gautier, qui avait joué un si mauvais tour à Flaubert! Ne lui avait-il pas chipé cette Égypte qui l'avait tant fait rêver? À la suite du romancier, l'Antiquité prit de nouvelles couleurs. Dont certaines seront bibliques grâce à Lewis Wallace.

\section{Ben-Hur ou l'Antiquité messianique (1880)}

Lewis Wallace (on dira ensuite Lew) est né le io avril I827 à Brookville, dans l'Indiana. Des études de droit le mènent au barreau mais, il s'engage, en i846-I847, dans la guerre que les États-Unis mènent contre le Mexique. Colonel au début de la guerre de Sécession, il s'y illustre en sauvant même Washington d'un coup de main ennemi. Major général à la fin de la guerre, il devient gouverneur du Nouveau Mexique de I878 à I88I (on peut même l'apercevoir dans un coin de western!), puis ambassadeur en Turquie de i88 à i885. Retiré alors de la vie politique, il continuera une carrière littéraire commencée en 1873 avec Le Dieu de beauté, un roman qui relate son expérience mexicaine et continuée avec le succès qu'on sait, en I88o, 
avec Ben-Hur. Un récit messianique. Il reviendra à l'Antiquité évangélique, en i885, avec Le Prince de l'Inde, un récit qui met en scène le Juif Errant. Il meurt en igo5.

Musicien, peintre, écrivain, soldat, diplomate, Lew Wallace avait tous les dons. Mais rien pourtant ne le prédisposait à écrire le roman qui le rendra universellement célèbre, Ben-Hur. Roman dont le héros, le prince Juda Ben-Hur, d'illustre famille, va voir son destin bouleversé. Capturé, humilié, dépouillé de son bien, envoyé aux galères, le jeune aristocrate doit se venger de son ancien ami, le Romain Messala. En chemin, il rencontrera l'amour et la révélation divine. Le sous-titre auquel on ne prête généralement assez peu d'attention indique bien le sens du récit: la venue d'un prophète nommé Jésus, fils de David, qui passait, aux yeux de certains Juifs, pour le Messie tant attendu.

Ce qui étonne dans l'accueil enthousiaste fait à Ben-Hur, à moins que ce ne soit un début d'explication, c'est la complète étrangeté de l'œuvre dans la production littéraire américaine de l'époque.Ben-Hur demeure, dans la littérature américaine de la fin du xix ${ }^{\mathrm{e}}$ siècle, littérature qui ne s'est jamais soucié d'Antiquité, un «apax». Peut-on en trouver la source en Europe? Rien n'est moins sûr: entre Fabiola et Quo Vadis? entre I854 et I895, rien, ou si peu, qui puisse, du moins dans le domaine romanesque, inspirer Wallace.

En revanche la science historique avait marché à grands pas et la figure de Jésus, dégagée des enluminures christiques, prenait, peu à peu, difficilement mais sûrement, épaisseur humaine. Dès ı835-ı836, D.F.Strauss, dans sa Vie de Jésus, tentait d'unir les faits historiques, à savoir l'existence d'un Jésus ancré dans une réalité historique, et les éléments mythiques ajoutés par la suite: double portrait d'un Jésus historique face à un Christ idéalisé. Sans avoir publié de vie de Jésus, F.C. Baur, dans son Paul apôtre de Jésus (1845) appliqua à l'histoire du christianisme primitif la fameuse triade hégélienne : thèse (le courant judéo-chrétien de Pierre et de Jacques), antithèse (le courant hellénistico-chrétien de Paul), synthèse (l'Église catholique). Sans nier toutefois l'existence de Jésus. Ce que finit par faire radicalement son adversaire, Bauer, qui voyait le christianisme primitif comme une fusion entre le stoïcisme et le judaïsme hellénistique. Cette thèse coûta à son auteur, en ı842, la défense d'enseigner.

Ce qui arriva, vingt ans plus tard, à E. Renan (I823-I892). Sa Vie de Jésus (I863) fut le premier pas de la critique française sur un terrain dont elle n'avait pas l'habitude et déclencha un tollé général et... un immense succès. Dix rééditions et onze traductions à la fin de l'année i863. L'année suivante, une version plus accessible, Jésus, se vend à un million d'exemplaires. En juin I864 Renan est révoqué de sa chaire d'hébreu du Collège de France, chaire qu'il ne retrouvera qu'en i87o. Historien, linguiste, archéologue, homme d'étude et de terrain, Renan, qui vient de publier en I874, son fameux Recueil des inscriptions sémitiques, ne pouvait pas ne pas être connu de Wallace. 
Mais ce sont surtout les avancées de la recherche archéologique qui ont dû retenir son attention: Américains, comme Edward Robinson qui découvre à Jérusalem ce qu'on nomme depuis «l'arche de Robinson », au pied du mont du Temple; Français, comme F. de Saulcy qui creuse, en I863, et met au jour, à l'extérieur de la vieille ville, des tombes royales; Anglais qui s'activent depuis la fondation, en I863, du “Palestine Exploration Fund » et dont l'un deux, Charles Warren, repère, entre I867 et I870, dans la cité de David, le «puits de Warren ». Bref, le roman de Wallace a les couleurs de l'actualité.

L'action relève à la fois de la parabole: un Juif va, peu à peu, ouvrir les yeux sur le Christ; qui plus est, un Juif de noble famille. Et du roman-feuilleton: la vengeance d'un homme dépouillé de ses biens et trahi par son meilleur ami. C'est ce mélange de ficelles romanesques et de ton inspiré qui donne au récit ce charme si particulier. La structure en est très classique et le découpage semble appeler déjà le scénariste! Des lieux mythiques: Jérusalem, Antioche, Rome, le désert. Des morceaux de bravoure: la bataille navale, la course de chars, la descente chez les lépreux. La figure de Jésus dont la main soulage la soif du prisonnier qui, à son tour, tentera ensuite de lui venir en aide. En vain, on s'en doute. Tout cela vaudra au roman un triomphal accueil. Il faudra attendre plus de vingt ans pour que, avec Freud, l’on revienne à Pompéi.

\section{Gradiva ou l'Antiquité fantasmatique (1903 et 1907)}

Depuis I896, Sigmund Freud a pris l'habitude de partir à l'étranger, la plupart du temps en Italie de la fin août à la mi-septembre. En I9o2, avec son frère Alexandre, il s'installe à Sorrente; il visite Naples et la région du Vésuve. L'étape la plus importante est, on s'en doute, Pompéi.

Freud possède des ouvrages sur la cité disparue. Il en a étudié les rues avec passion: ‘J'attends avidement les beaux jours; pendant les quelques semaines où j'ai pu avoir une heure de liberté, je n'ai rien fait qu'étudier les rues de Pompéi » (lettre à Fliess du ı2avril ı897). Il en a rêvé (lettre au même du 28 avril I897). Il a placé dans son bureau la reproduction d'un «fragment de mur pompéien avec un centaure et un faune» qui le “transporte vers son Italie tant désirée» (lettre au même du 24mai igor).

À Sorrente se mêlent dans son esprit le parfum des orangers et la beauté des ruines. Peu à peu, au cours de ses voyages en Italie et en Grèce, Freud va prendre conscience de l'étroite parenté qui existe entre l'archéologue et l'analyste. L'un et l'autre essaient d'exhumer ce qui est demeuré enfoui d'une antique civilisation ou d'un désir infantile.

Il aurait pu, s'il avait été plus amateur de fiction, ajouter l'auteur de romans historiques. Lui aussi, comme l'analyste, remonte dans le temps, pour retrouver 
dans le passé ce qui, comme le pensaient Gautier ou Waltari, est resté enseveli en lui-même; pour tenter, à travers des destins antiques, de comprendre le sien.

Durant l'été ıgo6, Freud lit une nouvelle de Wilhelm Jensen (I837-ıgII), parue en Igo3: Gradiva, fantaisie pompéienne, que Carl Jung lui a conseillée. Immédiatement il en écrit un commentaire, qui paraîtra en 1907: Le Délire et les rêves dans la Gradiva de Jensen. C'est qu'il a pris conscience qu'‘ il n'y a pas de meilleure analogie du refoulement - qui tout à la fois rend un élément psychique inaccessible et le conserve - qu'un ensevelissement comme celui qui a été le destin fatal de Pompéi et dont la ville a pu émerger à nouveau par le travail de la pioche». Cette approche ne rencontrera guère d'écho chez Jensen, qui refusera même de rencontrer Freud, mais elle ouvrira une voie féconde à l'application de la méthode analytique aux textes littéraires - et consacrera définitivement Pompéi dans son rôle de cité fantasmatique.

Freud, quant à lui, gardera toujours une tendresse secrète pour cette femme, la Gradiva. À Rome, en I907, il fait enfin sa connaissance: “Pense à ma joie, écrit-il à son épouse Martha, le 24 septembre, en rencontrant aujourd'hui au Vatican, après une si longue solitude, le visage connu d'un être qui m'est cher; mais la reconnaissance a été unilatérale, car il s'agissait de Gradiva, accrochée tout au haut d'un mur. Désormais le moulage du bas-relief montrant une jeune fille traversant une rue figurera dans son bureau - et dans celui de bien des analystes -, avec des fresques pompéiennes.

La nouvelle de Jensen, grâce à Freud, est devenue universellement célèbre. Son héros en est Norbert, un jeune archéologue qui croit retrouver à Pompéi une Pompéienne dont le bas relief l'a troublé: Gradiva. Il lui parle en latin, elle lui répond dans la même langue. Il comprendra à la fin du récit qu'en fait il avait retrouvé une amie d'enfance, bien oubliée depuis des années et dont il était secrètement épris.

Quel dommage que Freud n'ait pas connu Arria Marcella, souvenir de Pompéi, la nouvelle de Gautier déjà évoquée! Nul doute qu'il y aurait trouvé un plus beau sujet d'études. Car le récit de cette nuit fantastique où le jeune Octavien, en visite avec des amis à Pompéi, se retrouve dans la cité redevenue vivante, pourrait aisément faire l'objet d'une étude psychanalytique avec, bien sûr, toute la part d'arbitraire qu'elle suppose. Je verrais volontiers, pour ma part, dans le récit de Gautier, les quatre manifestations du désir et poserait les quatre termes de la phase fantasmatique: désir, interdit, transgression, sanction.

$\mathrm{Au}$ fond, si la cure analytique de Norbert a réussi : en bonne analyste, la pseudo Gradiva est entrée dans le fantasme de son analysant-amoureux, puis, par elle et pour elle, il en est sorti, pour revenir à la réalité. L'analyse d'Octavien, en revanche, a échoué: il n’a pas pu échapper au sien et il sera malheureux toute sa vie. N'en fut-il pas ainsi pour son créateur? 
On aurait pu aussi évoquer la Crète et les travaux d'Evans qui eurent un écho de l'œuvre de Nicos Kazantzakis ou l'Afrique du Sud avec les ruines de Zimbabwe qui firent rêver, en I885, H. Ridder Haggard et ses Mines du roi Salomon.

Ainsi donc, au siècle qui assiste à la naissance conjointe du roman historique et de l'archéologie, la pioche des archéologues a souvent inspiré la plume des romanciers. Sous les couleurs d'une Antiquité nouvelle, revivifiée, de nouveau vivante.

\section{Claude Aziza}

Université de la Sorbonne Nouvelle, Paris III

6, square de Port-Royal, 75013 Paris

claude.aziza@laposte.net 